\title{
Singular Riesz measures on symmetric cones
}

\author{
(Running title: Singular Riesz measures) \\ Abdelhamid Hassairi*, and Sallouha Lajmi \\ Sfax University, Tunisia.
}

\begin{abstract}
In this paper, we give an explicit description of a class of positive measures on symmetric cones defined by their Laplace transforms in the framework of the Riesz integrals. This work is motivated by the importance of these measures in probability theory and statistics since they represent a generalization of the measures generating the famous Wishart distributions.

Keywords: Jordan algebra, symmetric cone, generalized power, Laplace transform, Riesz measure.
\end{abstract}

AMS Classification : 46G12, 28A25.

\section{Introduction}

Many interesting results of analysis on Jordan algebras and their symmetric cones have been not only used as powerful mathematical tools but also as sources of inspiration in the development of other fields and more particularly of probability theory and Statistics. This seems to be due in particular to the importance, in certain areas of these fields, of the special case of the algebra of symmetric matrices and of its symmetric cone of positive definite matrices. For instance in 2001,

\footnotetext{
${ }^{*}$ Corresponding author. E-mail address: Abdelhamid.Hassairi@fss.rnu.tn
} 
Hassairi and Lajmi ([3]) have introduced a class of natural exponential families of probability distributions generated by measures related to the so-called Riesz integrals in analysis on symmetric cones (see [1], p.137). These measures and the generated probability distributions have been respectively called by these authors Riesz measures and Riesz probability distributions. The Riesz measures on a symmetric cone are in fact defined by their Laplace transforms in a fondamental theorem due to Gindikin ([2]). Roughly speaking, the theorem says that the generalized power $\Delta_{s}\left(\theta^{-1}\right)$ defined on a symmetric cone is the Laplace transform of a positive measure $R_{s}$ if and only if $s$ is in a given set $\Xi$ of $\mathbb{R}^{r}$, where $r$ is the rank of the algebra. When $s$ is in a well defined part of $\Xi$, the Riesz measure $R_{s}$ is absolutely continuous with respect to the Lebesgue measure on the symmetric cone and has a density which is expressed in terms of the generalized power. For the other elements $s$ of $\Xi$, the Riesz measure $R_{s}$ is concentrated on the boundary of the cone, we will say that they are singular Riesz measures. These measures were considered of complicated nature and their structure has never been explicitly determined although some among them have a probabilistic interpretation and play an important role in multivariate statistics. The aim of the present paper is to give an explicit description of the Riesz measure $R_{s}$ for all $s$ in $\Xi$. The question is very interesting from a mathematical point of view, in fact, besides the use of many important known facts from the analysis on symmetric cones, we have been led to develop many other useful results. On the other hand, we think that the knowledge of the way in which a singular Riesz measure is built should allow us to give a statistical interpretation of the generated family of probability distributions extending the one corresponding to the Wishart.

\section{Preliminaries}

In this section, we first recall some facts concerning Jordan algebras and their symmetric cones, for more details, we refer the reader to the book of Faraut and Korányi (1994),([1]) which is a complete and self-contained exposition on the subject. We then establish some new results on symmetric cones which we need in the description of the Riesz measures.

Recall that a Euclidean Jordan algebra is a Euclidean space $V$ with scalar product $\langle x, y\rangle$ and a bilinear map

$$
V \times V \rightarrow V, \quad(x, y) \longmapsto x . y
$$

called Jordan product such that, for all $x, y, z$ in $V$,

i) $x . y=y \cdot x$,

ii) $\langle x, y . z\rangle=\langle x . y, z\rangle$, 
iii) there exists $e$ in $V$ such that $e \cdot x=x$,

iv) $x \cdot\left(x^{2} \cdot y\right)=x^{2} \cdot(x \cdot y), \quad$ where we used the abbreviation $x^{2}=x \cdot x$.

A Euclidean Jordan algebra is said to be simple if it does not contain a nontrivial ideal. Actually to each Euclidean simple Jordan algebra, one attaches the set of Jordan squares

$$
\bar{\Omega}=\left\{x^{2} ; x \in V\right\} .
$$

Its interior $\Omega$ is a symmetric cone i.e. a cone which is

i) self dual, i.e., $\Omega=\{x \in V ; \quad\langle x, y\rangle>0 \quad \forall y \in \bar{\Omega} \backslash\{0\}\}$

ii) homogeneous, i.e. the subgroup $G(\Omega)$ of the linear group $G L(V)$ of linear automorphisms which preserve $\Omega$ acts transitively on $\Omega$.

iii) salient, i.e., $\Omega$ does not contain a line. Furthermore, it is irreducible in the sense that it is not the product of two cones.

Let now $x$ be in $V$. If $L(x)$ is the endomorphism of $V ; y \longmapsto x . y$ and $P(x)=2 L(x)^{2}-L\left(x^{2}\right)$, then $L(x)$ and $P(x)$ are symmetric for the Euclidean structure of $V$, the map $x \longmapsto P(x)$ is called the quadratic representation of $V$.

A element $c$ of $V$ is said to be idempotent if $c^{2}=c$, it is a primitive idempotent if furthermore $c \neq 0$ and is not the sum $t+u$ of two non null idempotents $t$ and $u$ such that $t . u=0$.

A Jordan frame is a set $\left\{c_{1}, c_{2}, \ldots . . c_{r}\right\}$ such that $\sum_{i=1}^{r} c_{i}=e$ and $c_{i} . c_{j}=\delta_{i, j} c_{i}$, for $1 \leq i, j \leq r$. It is an important result that the size $r$ of such a frame is a constant called the rank of $V$. For any element $x$ of a Euclidean simple Jordan algebra, there exists a Jordan frame $\left(c_{i}\right)_{1 \leq i \leq r}$ and $\left(\lambda_{1}, \ldots, \lambda_{r}\right) \in \mathbb{R}^{r}$ such that $x=\sum_{i=1}^{r} \lambda_{i} c_{i}$. The real numbers $\lambda_{1}, \lambda_{2}, \ldots, \lambda_{r}$ depend only on $x$, they are called the eigenvalues of $x$ and this decomposition is called its spectral decomposition. The trace and the determinant of $x$ are then respectively defined by $\operatorname{tr}(x)=\sum_{i=1}^{r} \lambda_{i}$ and $\operatorname{det} x=\prod_{i=1}^{r} \lambda_{i}$. If $c$ is a primitive idempotent of $V$, the only possible eigenvalues of $L(c)$ are $0, \frac{1}{2}$ and 1 . The corresponding spaces are respectively denoted by $V(c, 0), V\left(c, \frac{1}{2}\right)$ and $V(c, 1)$ and the decomposition

$$
V=V(c, 1) \oplus V\left(c, \frac{1}{2}\right) \oplus V(c, 0)
$$

is called the Peirce decomposition of $V$ with respect to $c$. An element $x$ of $V$ can then be written in a unique way as

$$
x=x_{1}+x_{12}+x_{0}
$$


with $x_{1}$ in $V(c, 1), x_{12}$ in $V\left(c, \frac{1}{2}\right)$ and $x_{0}$ in $V(c, 0)$, which is also called the Peirce decomposition of $x$ with respect to the idempotent $c$. We will denote $\Omega_{c}$ the symmetric cone associated to the sub-algebra $V(c, 1)$ and $\operatorname{det}_{c}$ the determinant in this sub-algebra.

Suppose now that $\left(c_{i}\right)_{1 \leq i \leq r}$ is a Jordan frame in $V$ and, for $1 \leq i, j \leq r$, we set

$$
V_{i j}= \begin{cases}V\left(c_{i}, 1\right)=\mathbb{R} c_{i} & \text { if } i=j \\ V\left(c_{i}, \frac{1}{2}\right) \cap V\left(c_{j}, \frac{1}{2}\right) & \text { if } i \neq j\end{cases}
$$

Then (See [1], Th.IV.2.1) we have the Peirce decomposition $V=\bigoplus_{i \leq j} V_{i j}$ with respect to the Jordan frame $\left(c_{i}\right)_{1 \leq i \leq r}$. The dimension of $V_{i j}$ is, for $i \neq j$, a constant $d$ called the Jordan constant, it is related to the dimension $n$ and the rank $r$ of $V$ by the relation $n=r+\frac{d}{2} r(r-1)$.

For $1 \leq k \leq r$, we have

$$
V\left(c_{1}+\ldots+c_{k}, 1\right)=\bigoplus_{i \leq j \leq k} V_{i j}, V\left(c_{1}+\ldots+c_{k}, \frac{1}{2}\right)=\bigoplus_{1 \leq i \leq k<j} V_{i j}
$$

In the following proposition, we establish some useful intermediary results.

Proposition 2.1 Let $c$ be an idempotent of $V$. Then

i) $\Omega_{c}=P(c)(\Omega)$

ii) for all $x$ in $V(c, 1), 2 L(x)_{\mid V\left(c, \frac{1}{2}\right)}$ is an endomorphism of $V\left(c, \frac{1}{2}\right)$ with determinant equal to $\operatorname{det}_{c}(x)^{d(r-k)}$, where $k$ is the rank of $c$

iii) if $x$ in $V(c, 1)$ is invertible, then $2 L(x)_{\mid V\left(c, \frac{1}{2}\right)}$ is an automorphism of $V\left(c, \frac{1}{2}\right)$ with inverse equal to $2 L\left(x^{-1}\right)_{\mid V\left(c, \frac{1}{2}\right)}$.

iv) for all $x$ in $V(c, 1), L\left(x^{2}\right)_{\mid V\left(c, \frac{1}{2}\right)}=\frac{1}{2} L\left(x^{2}\right)_{\mid V\left(c, \frac{1}{2}\right)}$

\section{Proof.}

i) From Theorem III.2.1 in [1], we have that the symmetric cone of a Jordan algebra is the set of element $x$ in $V$ for which $L(x)$ is positive definite.

Let $x$ be in $\Omega$. For $y \in V(c, 1), y \neq 0$, we have:

$$
\begin{aligned}
\langle L(P(c) x)(y), y\rangle & =\langle P(c)(x) y, y\rangle \\
& =\left\langle P(c)(x), y^{2}\right\rangle \\
& =\left\langle x, P(c) y^{2}\right\rangle \\
& =\langle y, x y\rangle>0
\end{aligned}
$$

Thus $P(c) \Omega \subseteq \Omega_{c}$.

Now, let $w \in \Omega_{c}$, then $w+(e-c)$ is an element of $\Omega$. Since $P(c)(w+(e-c))=$ $P(c)(w)=w$, we obtain that $\Omega_{c} \subseteq P(c) \Omega$. 
ii) Let $x \in V(c, 1)$. It is known (see Faraut-Korányi, Prop IV.1.1) that $V(c, 1) . V\left(c, \frac{1}{2}\right) \subseteq V\left(c, \frac{1}{2}\right)$, hence $2 L(x)_{\mid V\left(c, \frac{1}{2}\right)}$ is an endomorphism of $V\left(c, \frac{1}{2}\right)$.

As $c$ is an idempotent of rank $k$, there exit $c_{1}, c_{2}, \ldots, c_{k}$ orthogonal idempotents and $\left(\lambda_{1}, \ldots, \lambda_{k}\right) \in \mathbb{R}^{k}$ such that $c=\sum_{i=1}^{k} c_{i}$ and $x=\sum_{i=1}^{k} \lambda_{i} c_{i}$, so that $\operatorname{det}_{c} x=\prod_{i=1}^{k} \lambda_{i}$. Similarly, since $e-c$ is an idempotent with rank $r-k$, there exit $c_{k+1}, c_{k+2}, \ldots, c_{r}$ orthogonal idempotents such that $e-c=\sum_{i=1}^{r-k} c_{k+i}$. The system $\left(c_{i}\right)_{1 \leq i \leq r}$ is a Jordan frame of $V$. If for $1 \leq i \leq k$, we set $V_{i, k+1}=\bigoplus_{j=k+1}^{r} V_{i j}$, then $V\left(c, \frac{1}{2}\right)=\bigoplus_{i=1}^{k} V_{i, k+1}$. We can easily show that $2 L(x)_{\mid V_{i, k+1}}=\lambda_{i} I d_{i, k+1}$, where $I d_{i, k+1}$ is the identity on the space $V_{i, k+1}$. As the dimension of $V_{i, k+1}$ is equal to $(r-k) d$, we have that the determinant of $2 L(x)_{\mid V\left(c, \frac{1}{2}\right)}$ is equal to $\prod_{i=1}^{k} \lambda_{i}^{(r-k) d}=\left(\operatorname{det}_{c} x\right)^{(r-k) d}$.

iii) If $x$ is invertible in $V(c, 1)$, then $\lambda_{1}, \ldots, \lambda_{k}$ are different from zero and $x^{-1}=\sum_{p=1}^{k} \lambda_{p}^{-1} c_{p}$. Therefore, $2 L(x)_{\mid V_{i, k+1}}$ is an automorphism of $V_{i, k+1}$ with inverse $\lambda_{i}^{-1} I d_{i, k+1}$ and it follows that $2 L(x)_{\mid V\left(c, \frac{1}{2}\right)}$ is an automorphism of $V\left(c, \frac{1}{2}\right)$ with inverse $2 L\left(x^{-1}\right)_{\mid V\left(c, \frac{1}{2}\right)}$.

iv) We have that $x^{2}=\sum_{p=1}^{k} \lambda_{p}^{2} c_{p}$ and for all $1 \leq i \leq k, 2 L(x)_{\mid V_{i, k+1}}=\lambda_{i} I d_{i, k+1}$. Then

$$
L(x)_{\mid V_{i, k+1}}^{2}=\frac{1}{4} \lambda_{i}^{2} I d_{i, k+1}=\frac{1}{2} \frac{\lambda_{i}^{2}}{2} I d_{i, k+1}=\frac{1}{2} L\left(x^{2}\right)_{\mid V_{i, k+1}} .
$$

Thus, we conclude that $L\left(x^{2}\right)_{\mid V\left(c, \frac{1}{2}\right)}=\frac{1}{2} L\left(x^{2}\right)_{\mid V\left(c, \frac{1}{2}\right)}$.

Besides, the results shown above, we will use the facts stated in the following proposition due to Massam and Neher ([6]).

Proposition 2.2 Let $c$ be an idempotent of $V, u_{1}$ in $V(c, 1), v_{12}$ in $V\left(c, \frac{1}{2}\right)$, and $u_{0}, z_{0}$ in $V(c, 0)$. Then

i) $\left\langle u_{1}, P\left(v_{12}\right) z_{0}\right\rangle=2\left\langle v_{12}, L\left(z_{0}\right) L\left(u_{1}\right) v_{12}\right\rangle$

ii) $L\left(z_{0}\right) L\left(u_{1}\right)=L\left(u_{1}\right) L\left(z_{0}\right)$ 
iii) If $u_{1} \in \Omega_{c}$ and $z_{0} \in \Omega_{e-c}$, then $L\left(u_{1}\right) L\left(z_{0}\right)_{\mid V\left(c, \frac{1}{2}\right)}$ is a positive definite endomorphism.

Throughout, we suppose that the Jordan frame $\left(c_{i}\right)_{1 \leq i \leq r}$ is fixed in $V$. For $1 \leq k \leq r$, let $P_{k}$ denote the orthogonal projection on the Jordan subalgebra

$$
V^{(k)}=V\left(c_{1}+c_{2}+\ldots+c_{k}, 1\right),
$$

$\operatorname{det}^{(k)}$ the determinant in the subalgebra $V^{(k)}$ and, for $x$ in $V, \Delta_{k}(x)=\operatorname{det}^{(k)}\left(P_{k}(x)\right)$. The real number $\Delta_{k}(x)$ is called the principal minor of order $k$ of $x$ with respect to the frame $\left(c_{i}\right)_{1 \leq i \leq r}$.

The generalized power with respect to the Jordan frame $\left(c_{i}\right)_{1 \leq i \leq r}$ is the polynomial function defined in $x$ of $V$ by

$$
\Delta_{s}(x)=\Delta_{1}(x)^{s_{1}-s_{2}} \Delta_{2}(x)^{s_{2}-s_{3}} \ldots \Delta_{r}(x)^{s_{r}} .
$$

Note that $\Delta_{s}(x)=(\operatorname{det}(x))^{p}$ if $s=(p, p, \ldots, p)$ with $p \in \mathbb{R}$, and if $x=\sum_{i=i}^{r} \lambda_{i} c_{i}$, then $\Delta_{s}(x)=\lambda_{1}^{s_{1}} \lambda_{2}^{s_{2}} \ldots \lambda_{r}^{s_{r}}$. It is also easy to see that $\Delta_{s+s^{\prime}}(x)=\Delta_{s}(x) \cdot \Delta_{s}(x)$. In particular, if $m \in \mathbb{R}$ and $s+m=\left(s_{1}+m, s_{2}+m, \ldots \ldots, s_{r}+m\right)$, we have $\Delta_{s+m}(x)=\Delta_{s}(x) \operatorname{det}(x)^{m}$.

Now for the fixed Jordan frame $\left(c_{i}\right)_{1 \leq i \leq r}$, and for $1 \leq l \leq r$ we define

$$
\sigma_{l}=\sum_{i=1}^{l} c_{i}
$$

and we suppose that $V\left(\sigma_{l}, 1\right)$ and $V\left(e-\sigma_{l}, 1\right)$ are respectively equipped with the Jordan frames $\left(c_{i}\right)_{1 \leq i \leq l}$ and $\left(c_{i}\right)_{l+1 \leq i \leq r}$. Then we have the following result which allows the calculation of the general power of some projections. For the proof we refer the reader to Hassairi and Lajmi ([4]).

Theorem 2.3 Let $1 \leq l \leq r-1$, and denote $\theta_{0}$ the orthogonal projection of an element $\theta$ of the cone $\Omega$ on $V\left(e-s_{l}, 1\right)$. Then

i) $\Delta_{l}\left(\theta^{-1}\right)=\operatorname{det}\left(\theta^{-1}\right) \operatorname{det}_{e-\sigma_{l}}\left(\theta_{0}\right)$,

ii) for $l+1 \leq k \leq r-1, \frac{\Delta_{k+1}\left(\theta^{-1}\right)}{\Delta_{k}\left(\theta^{-1}\right)}=\frac{\Delta_{k+1-l}^{e-\sigma_{l}}\left(\theta_{0}^{-1}\right)}{\Delta_{k-l}^{e-\sigma_{l}}\left(\theta_{0}^{-1}\right)}$, and $\frac{\Delta_{l+1}\left(\theta^{-1}\right)}{\Delta_{l}\left(\theta^{-1}\right)}=\Delta_{1}^{e-\sigma_{l}}\left(\theta_{0}^{-1}\right)$.

We now introduce the set $\Xi$ of elements $s=\left(s_{1}, \ldots, s_{r}\right)$ in $\mathbb{R}^{r}$ defined in the following way:

For a given real number $u \geq 0$, we set

$$
\varepsilon(u)=0 \quad \text { if } \quad u=0
$$




$$
\varepsilon(u)=1 \quad \text { if } \quad u>0
$$

Given $u=\left(u_{1}, \ldots, u_{r}\right) \in \mathbb{R}_{+}^{r}$, we define

$$
s_{1}=u_{1} \text { and } s_{i}=u_{i}+\frac{d}{2}\left(\varepsilon\left(u_{1}\right)+\ldots+\varepsilon\left(u_{i-1}\right)\right) \text { for } \quad 2 \leq i \leq r .
$$

Note that the set $\Xi$ contains $\left.\prod_{i=1}^{r}\right](i-1) \frac{d}{2},+\infty[$, and that

$$
\left.\Lambda=\{p \in \mathbb{R} \text { such that }(p, \ldots, p) \in \Xi\}=\left\{\frac{d}{2}, \ldots, \frac{d}{2}(r-1)\right\} \cup\right](r-1) \frac{d}{2},+\infty[
$$

The definition of the Riesz measure is based on the following theorem due to Gindikin ([2]), for a proof we refer the reader to Faraut and Korányi (1994). The Laplace transform of a positive measure $\mu$ on $V$ is defined by

$$
L_{\mu}(\theta)=\int_{V} \exp (\langle\theta, x\rangle) \mu(d x) .
$$

Theorem 2.4 There exists a positive measure $R_{s}$ on $V$ with Laplace transform defined on $-\Omega$ by $L_{R_{s}}(\theta)=\Delta_{s}\left(-\theta^{-1}\right)$ if and only if $s$ is in the set $\Xi$.

Hassairi and Lajmi ([3]) have called the measure $R_{s}$ Riesz measure and they have used it to introduce a class of probability distributions which is an important extension of the famous Wishart ones. When $s=\left(s_{1}, s_{2}, \ldots . ., s_{r}\right)$ is in $\left.\prod_{i=1}^{r}\right](i-$ 1) $\frac{d}{2},+\infty$ [, the measure $R_{s}$ has an explicit expression. In fact, if for $s$ such that for all $i, s_{i}>(i-1) \frac{d}{2}$, we consider the measure

$$
R_{s}=\frac{1}{\Gamma_{\Omega}(s)} \Delta_{s-\frac{n}{r}}(x) \mathbf{1}_{\Omega}(x) d x
$$

where $\Gamma_{\Omega}(s)=(2 \pi)^{\frac{n-r}{2}} \prod_{j=1}^{r} \Gamma\left(s_{j}-(j-1) \frac{d}{2}\right)$, then it is proved in Faraut Korányi (1994), Theo. VII.1.2, that the Laplace transform of $R_{s}$ is equal to $\Delta_{s}\left(-\theta^{-1}\right)$ for $\theta \in-\Omega$, that is for all $\theta \in-\Omega$,

$$
\frac{1}{\Gamma_{\Omega}(s)} \int \exp (\langle\theta, x\rangle) \Delta_{s-\frac{n}{r}}(x) \mathbf{1}_{\Omega}(x)(d x)=\Delta_{s}\left(-\theta^{-1}\right) .
$$

\section{Description of the Riesz measures}

In this section, we give a complete description of the Riesz mesure $R_{s}$ inclosing the ones corresponding to $s$ in $\left.\Xi \backslash \prod_{i=1}^{r}\right](i-1) \frac{d}{2},+\infty[$ which are concentrated on the boundary $\partial \Omega$ of the symmetric cone $\Omega$. In order to do so, we need to recall 
some facts on the boundary structure of the cone $\Omega$. More precisely, we have the following useful decomposition of the closed cone $\bar{\Omega}$ into orbits under the action of the group $G$, connected component of the identity in $G(\Omega)$, which appears in Lasalle (1987) and in Faraut and Korányi (1994). Recall that for the fixed Jordan frame $\left(c_{i}\right)_{1 \leq i \leq r}$ and $1 \leq l \leq r, \sigma_{l}=\sum_{i=1}^{l} c_{i}$.

Proposition 3.1 i) Let $x$ be in $\bar{\Omega}$. Then $x$ is of rank $l$ if and only if $x \in G \sigma_{l}$

ii) We have that $\bar{\Omega}=\bigcup_{l=1}^{r} G \sigma_{l}$.

More precisely, $\Omega=G \sigma_{r}=G e$ and $\partial \Omega=\bigcup_{l=1}^{r-1} G \sigma_{l}$

iii) Denote for $1 \leq l \leq r-1$

$$
J_{l}=\left\{x \in G \sigma_{l} ; \Delta_{l}(x) \neq 0\right\} .
$$

then $J_{l}$ is an open subset dense in $G \sigma_{l}$.

iv)Suppose that $x=x_{1}+x_{12}+x_{0}$ is the Peirce decomposition of $x$ with respect to $\sigma_{l}$, then the map

$$
\Omega_{\sigma_{l}} \times V\left(\sigma_{l}, \frac{1}{2}\right) \rightarrow J_{l} \quad ; \quad\left(x_{1}, x_{12}\right) \longmapsto x_{1}+x_{12}+2\left(e-\sigma_{l}\right)\left[x_{12}\left(x_{1}^{-1} x_{12}\right)\right]
$$

is a bijection.

As a corollary of the last point, we have that an element $x$ of $J_{l}$ can be written in a unique way as $x=x_{1}+x_{12}+\left(e-\sigma_{l}\right) v^{2}$, where $v=\frac{1}{2} \sqrt{x_{1}^{-1}} x_{12}$.

We now give the description of the Riesz measures $R_{s}$ when $s$ has a particular form, we then give the general case.

Theorem 3.2 Let $l$ be in $\{1, \ldots, r\}, \sigma_{l}=\sum_{i=1}^{l} c_{i}$, and $u=\left(u_{1}, \ldots, u_{l}\right)$ in $\mathbb{R}^{l}$ such that $u_{i}>(i-1) \frac{d}{2}$, for $1 \leq i \leq l$. Consider the measure

$$
\gamma_{l}\left(d x_{1}, d v\right)=\frac{\Delta_{u}^{\sigma_{l}}\left(x_{1}\right)\left(\operatorname{det}_{\sigma_{l}}\left(x_{1}\right)\right)^{-1-(l-1) \frac{d}{2}}}{(2 \pi)^{l(r-l) \frac{d}{2}} \Gamma_{\Omega_{\sigma_{l}}}(u)} \mathbf{1}_{\Omega_{\sigma_{l}} \times V\left(\sigma_{l}, \frac{1}{2}\right)}\left(x_{1}, v\right) d x_{1} d v
$$

and the map

$$
\alpha: \Omega_{\sigma_{l}} \times V\left(\sigma_{l}, \frac{1}{2}\right) \rightarrow V \quad ; \quad\left(x_{1}, v\right) \longmapsto x_{1}+2 v \sqrt{x_{1}}+\left(e-\sigma_{l}\right) v^{2} .
$$


Then the Laplace transform of the image $\mu_{l}=\alpha \gamma_{l}$ of $\gamma_{l}$ by $\alpha$ is defined on $-\Omega$ and is given by

$$
L_{\mu_{l}}(\theta)=\Delta_{s}\left(-\theta^{-1}\right),
$$

where $s=\left(u_{1}, \ldots, u_{l}, \frac{d l}{2}, \ldots, \frac{d l}{2}\right) \in \mathbb{R}^{r}$.

Proof. Let $\theta$ be in $-\Omega$ and let $\theta=\theta_{1}+\theta_{12}+\theta_{0}$ be its Peirce decomposition with respect to $\sigma_{l}$. Then according to Proposition 2.1, i), we have that $\theta_{1}=P\left(\sigma_{l}\right)(\theta)$ is in $-\Omega_{\sigma_{l}}$ and $\theta_{0}=P\left(e-\sigma_{l}\right)(\theta)$ is in $-\Omega_{e-\sigma_{l}}$. Let us calculate the Laplace transform of $\mu_{l}$ in $\theta$.

$$
\begin{aligned}
L_{\mu_{l}}(\theta)= & \int_{\Omega_{\sigma_{l}} \times V\left(\sigma_{l}, \frac{1}{2}\right)} \exp \left(\left\langle\theta, \alpha\left(x_{1}, v\right)\right\rangle \gamma_{l}\left(d x_{1}, d v\right)\right. \\
= & \int_{\Omega_{\sigma_{l}} \times V\left(\sigma_{l}, \frac{1}{2}\right)} \exp \left(\left\langle\theta_{1}, x_{1}\right\rangle+\left\langle\theta_{12}, 2 v \sqrt{x_{1}}\right\rangle+\left\langle\theta_{0}, v^{2}\right\rangle\right) \\
& \frac{\Delta_{u}^{\sigma_{l}}\left(x_{1}\right) \operatorname{det}_{\sigma_{l}}\left(x_{1}\right)^{-1-(l-1) \frac{d}{2}}}{(2 \pi)^{l(r-l) \frac{d}{2}} \Gamma_{\Omega_{\sigma_{l}}}(u)} d x_{1} d v
\end{aligned}
$$

This may be written as

$$
L_{\mu_{l}}(\theta)=\int_{\Omega_{\sigma_{l}}} I\left(x_{1}\right) \exp \left(\left\langle\theta_{1}, x_{1}\right\rangle\right) \Delta_{u}^{\sigma_{l}}\left(x_{1}\right) \operatorname{det}_{\sigma_{l}}\left(x_{1}\right)^{-1-(l-1) \frac{d}{2}} \frac{d x_{1}}{\Gamma_{\Omega_{\sigma_{l}}}(u)},
$$

where

$$
I\left(x_{1}\right)=\frac{1}{(2 \pi)^{l(r-l) \frac{d}{2}}} \int_{V\left(\sigma_{l}, \frac{1}{2}\right)} \exp \left(\left\langle\theta_{12}, 2 v \sqrt{x_{1}}\right\rangle+\left\langle\theta_{0}, v^{2}\right\rangle\right) d v .
$$

According to Prop 2.2, iii) and Prop 2.1, iii), we have that $2 L\left(-\theta_{0}\right)_{\mid V\left(\sigma_{l}, \frac{1}{2}\right)}=$ $L\left(4 \sigma_{l}\right) L\left(-\theta_{0}\right)_{\mid V\left(\sigma_{l}, \frac{1}{2}\right)}$ is an automorphism of $V\left(\sigma_{l}, \frac{1}{2}\right)$ whose the inverse is equal to $2 L\left(-\theta_{0}^{-1}\right)_{\mid V\left(\sigma_{l}, \frac{1}{2}\right)}$. Thus, one can write

$$
I\left(x_{1}\right)=\frac{1}{(2 \pi)^{l(r-l) \frac{d}{2}}} \int_{V\left(\sigma_{l}, \frac{1}{2}\right)} \exp \left(\left\langle 2 \sqrt{x_{1}} \theta_{12}, v\right\rangle-\frac{1}{2}\left\langle 2 L\left(-\theta_{0}\right) v, v\right\rangle\right) d v .
$$

Using Lemma VII.2.5 Faraut and Korányi (1994), then again Proposition 2.1, we get

$$
\begin{aligned}
I\left(x_{1}\right) & =\left(\operatorname{det} 2 L\left(-\theta_{0}^{-1}\right)_{\mid V\left(\sigma_{l}, \frac{1}{2}\right)}\right)^{\frac{1}{2}} \exp \left(\frac{1}{2}\left\langle 2 \sqrt{x_{1}} \theta_{12}, 2 L\left(-\theta_{0}^{-1}\right) 2 \sqrt{x_{1}} \theta_{12}\right\rangle\right) \\
& =\operatorname{det}_{e-\sigma_{l}}\left(-\theta_{0}^{-1}\right)^{l \frac{d}{2}} \exp \left(\frac{1}{2}\left\langle 2 \sqrt{x_{1}} \theta_{12}, 4 L\left(-\theta_{0}^{-1}\right) L\left(\sqrt{x_{1}}\right) \theta_{12}\right\rangle\right) .
\end{aligned}
$$


As $L\left(\sqrt{x_{1}}\right)$ is symmetric, we can write

$$
I\left(x_{1}\right)=\operatorname{det}_{e-\sigma_{l}}\left(-\theta_{0}^{-1}\right)^{l \frac{d}{2}} \exp \left(\left\langle 4 \theta_{12}, L\left(\sqrt{x_{1}}\right)^{2} L\left(-\theta_{0}^{-1}\right) \theta_{12}\right\rangle\right) .
$$

Proposition 2.1 implies that

$$
I\left(x_{1}\right)=\operatorname{det}_{e-\sigma_{l}}\left(-\theta_{0}^{-1}\right)^{l \frac{d}{2}} \exp \left(2\left\langle\theta_{12}, L\left(x_{1}\right) L\left(-\theta_{0}^{-1}\right) \theta_{12}\right\rangle\right) .
$$

Finally, from Proposition 2.2, we deduce that

$$
I\left(x_{1}\right)=\operatorname{det}_{e-\sigma_{l}}\left(-\theta_{0}^{-1}\right)^{l \frac{d}{2}} \exp \left(\left\langle x_{1}, P\left(\theta_{12}\right)\left(-\theta_{0}^{-1}\right)\right\rangle\right) .
$$

Now inserting this in (3), we obtain

$$
\begin{aligned}
L_{\mu_{l}}(\theta)= & \frac{\operatorname{det}_{e-\sigma_{l}}\left(-\theta_{0}\right)^{-l \frac{d}{2}}}{\Gamma_{\Omega_{\sigma_{l}}}(u)} \int_{\Omega_{\sigma_{l}}} \exp \left(\left\langle x_{1}, \theta_{1}-P\left(\theta_{12}\right)\left(\theta_{0}^{-1}\right)\right\rangle\right) \Delta_{u}^{\sigma_{l}}\left(x_{1}\right) \\
& \quad \operatorname{det}_{\sigma_{l}}\left(x_{1}\right)^{-1-(l-1) \frac{d}{2}} d x_{1} \\
= & \operatorname{det}_{e-\sigma_{l}}\left(-\theta_{0}\right)^{-l \frac{d}{2}} \Delta_{u}^{\sigma_{l}}\left(-\left(\theta_{1}-P\left(\theta_{12}\right)\left(\theta_{0}^{-1}\right)\right)^{-1}\right) .
\end{aligned}
$$

Since $\left(\theta^{-1}\right)_{l}=P\left(\sigma_{l}\right)\left(\theta^{-1}\right)=\left(\theta_{1}-P\left(\theta_{12}\right)\left(\theta_{0}^{-1}\right)\right)^{-1}$ and according to Theorem 2.3 , we can write

$$
\begin{aligned}
L_{\mu_{l}}(\theta) & =\operatorname{det}_{e-\sigma_{l}}\left(-\theta_{0}\right)^{-l \frac{d}{2}} \Delta_{u}^{\sigma_{l}}\left(-\left(\theta^{-1}\right)_{l}\right) \\
& =\left(\frac{\Delta_{l}\left(-\theta^{-1}\right)}{\operatorname{det}\left(-\theta^{-1}\right)}\right)^{-l \frac{d}{2}} \Delta_{u}^{\sigma_{l}}\left(-\left(\theta^{-1}\right)_{l}\right),
\end{aligned}
$$

Therefore,

$$
\begin{aligned}
L_{\mu_{l}}(\theta) & =\Delta_{1}\left(-\theta^{-1}\right)^{u_{1}-u_{2}} \ldots \Delta_{l-1}\left(-\theta^{-1}\right)^{u_{l-1}-u_{l}} \Delta_{l}\left(-\theta^{-1}\right)^{u_{l}-l \frac{d}{2}} \operatorname{det}\left(-\theta^{-1}\right)^{l \frac{d}{2}} \\
& =\Delta_{s}\left(-\theta^{-1}\right)
\end{aligned}
$$

where $s=\left(u_{1}, \ldots, u_{l}, l \frac{d}{2}, \ldots, l \frac{d}{2}\right)$ in $\mathbb{R}^{r}$

corollary 3.3 For $1 \leq l \leq r-1$, the measure $\mu_{l}$ is concentrated on the boundary $\partial \Omega$ of the symmetric cone $\Omega$.

Proof. In fact, $\mu_{l}$ is concentrated on on the set $J_{l}=\left\{x \in G \sigma_{l} ; \Delta_{l}(x) \neq 0\right\}$ which is dense in $G_{\sigma_{l}}($ prop 3.1)). 
Theorem 3.4 Let $l$ be in $\{1, \ldots, r-1\}$, and suppose that for $u=\left(u_{1}, \ldots, u_{r-l}\right) \in$ $\mathbb{R}_{+}^{r-l}$, there exists a measure $\mu_{u}$ on $V\left(e-\sigma_{l}, 1\right)$ such that the Laplace transform is defined on $-\Omega_{e-\sigma_{l}}$ and is equal to $\Delta_{u}^{e-\sigma_{l}}\left(-\theta_{0}^{-1}\right)$. Then the Laplace transform of the measure $\mu$ image of $\mu_{u}$ by the injection of $V\left(e-\sigma_{l}, 1\right)$ into $V$ is defined on $-\Omega$ and $L_{\mu}(\theta)=\Delta_{s}\left(-\theta^{-1}\right)$, where $s=\left(0, \ldots 0, u_{1}, \ldots, u_{r-l}\right) \in \mathbb{R}_{+}^{r}$.

Proof. Let $x=x_{1}+x_{12}+x_{0}$ and $\theta=\theta_{1}+\theta_{12}+\theta_{0}$ be respectively the Peirce decomposition with respect to $\sigma_{l}$ of an element $x$ of $V$ and an element $\theta$ of $-\Omega$. Then

$$
\begin{aligned}
L_{\mu}(\theta) & =\int_{V\left(e-\sigma_{l}, 1\right)} \exp \left(\left\langle\theta, x_{0}\right\rangle\right) \mu_{u}\left(d x_{0}\right) \\
& =\int_{V\left(e-\sigma_{l}, 1\right)} \exp \left(\left\langle\theta_{0}, x_{0}\right\rangle\right) \mu_{u}\left(d x_{0}\right) \\
& =\Delta_{u}^{e-\sigma_{l}}\left(-\theta_{0}^{-1}\right) \\
& =\Delta_{1}^{e-\sigma_{l}}\left(-\theta_{0}^{-1}\right)^{u_{1}}\left(\frac{\Delta_{2}^{e-\sigma_{l}}\left(-\theta_{0}^{-1}\right)}{\Delta_{1}^{e-\sigma_{l}}\left(-\theta_{0}^{-1}\right)}\right)^{u_{2}} \ldots\left(\frac{\Delta_{r-l}^{e-\sigma_{l}}\left(-\theta_{0}^{-1}\right)}{\Delta_{r-l-1}^{e-\sigma_{l}}\left(-\theta_{0}^{-1}\right)}\right)^{u_{r-l}}
\end{aligned}
$$

This according to Theorem 2.3 leads to

$$
\begin{aligned}
L_{\mu}(\theta) & =\left(\frac{\Delta_{l+1}\left(-\theta^{-1}\right)}{\Delta_{l}\left(-\theta^{-1}\right)}\right)^{u_{1}}\left(\frac{\Delta_{l+2}\left(-\theta^{-1}\right)}{\Delta_{l+1}\left(-\theta^{-1}\right)}\right)^{u_{2}} \ldots\left(\frac{\Delta_{r}\left(-\theta^{-1}\right)}{\Delta_{r-1}\left(-\theta^{-1}\right)}\right)^{u_{r-l}} \\
& =\Delta_{l}\left(-\theta^{-1}\right)^{-u_{1}} \Delta_{l+1}\left(-\theta^{-1}\right)^{u_{1}-u_{2}} \ldots \Delta_{r}\left(-\theta^{-1}\right)^{u_{r-l}} \\
& =\Delta_{s}\left(-\theta^{-1}\right)
\end{aligned}
$$

where $s=\left(0, \ldots, 0, u_{1}, \ldots, u_{r-l}\right) \in \mathbb{R}_{+}^{r}$.

We come now to the construction of the Riesz measure $R_{s}$ for any $s=\left(s_{1}, \ldots, s_{r}\right)$ in the set $\Xi$. From the definition of $\Xi$, there exists $u=\left(u_{1}, \ldots, u_{r}\right) \in \mathbb{R}_{+}^{r}$ such that

$$
s_{1}=u_{1} \text { and } s_{i}=u_{i}+\frac{d}{2}\left(\varepsilon\left(u_{1}\right)+\ldots+\varepsilon\left(u_{i-1}\right)\right) .
$$

We will use $\left(u_{1}, \ldots, u_{r}\right)$, to construct a partition $\left(A_{i}\right)$ of the set $\{1, \ldots, r\}$ such that, for all $i$, we have either $u_{j}=0, \forall j \in A_{i}$ or $u_{j}>0, \forall j \in A_{i}$. Such a partition is important in the description of the measure $R_{s}$.

Consider the sequences of integers $i_{1}, \ldots, i_{k}$ and $j_{1}, \ldots, j_{k}$ built as follows:

$i_{1}=\inf \left\{p \geq 0\right.$ such that $\left.u_{p+1} \neq 0\right\}$,

$j_{l}=\inf \left\{p \geq 0\right.$ such that $\left.u_{i_{l}+p+1}=0\right\}, 1 \leq l \leq k$,

$i_{l}=\inf \left\{p \geq i_{l-1}+j_{l-1}\right.$ such that $\left.u_{p+1} \neq 0\right\}, 2 \leq l \leq k$, 
In this way, we get a partition of $u=\left(u_{1}, \ldots, u_{r}\right)$ in the form:

$u=(\underbrace{0, \ldots, 0}_{i_{1} \text { terms }}, \underbrace{u_{i_{1}+1}, \ldots, u_{i_{1}+j_{1}}}_{j_{1} \text { terms }}, \ldots, \underbrace{0, \ldots, 0}_{j_{l} \text { terms }}, \underbrace{u_{i_{l}}, \ldots, u_{i_{l}+j_{l}}}_{i_{l}+1}, \ldots, \underbrace{0, \ldots, 0}_{\text {terms }}, \underbrace{u_{i_{k}}, \ldots, u_{i_{k}+j_{k}}}_{i_{k}+1}, \ldots)$.

This partition of $u$ leads to the following partition of the set $\{1, \ldots, r\}$ defined by

$$
\begin{gathered}
I_{0}^{\prime}= \begin{cases}\emptyset & \text { if } \quad i_{1}=0 \\
\left\{1, \ldots, i_{1}\right\} & \text { if } \quad i_{1} \neq 0\end{cases} \\
I_{l}^{\prime}=\left\{i_{l}+j_{l}+1, \ldots, i_{l+1}\right\} \quad \text { if } \quad 1 \leq l \leq k-1 . \\
I_{k}^{\prime}= \begin{cases}\emptyset & \text { if } \quad i_{k}+j_{k}=r \\
\left\{i_{k}+j_{k}+1, \ldots, r\right\} & \text { if } \quad i_{k}+j_{k}<r\end{cases}
\end{gathered}
$$

and

$$
I_{l}=\left\{i_{l}+1, \ldots, i_{l}+j_{l}\right\} \quad \text { if } \quad 1 \leq l \leq k
$$

Thus we have that

$$
\bigcup_{1 \leq p \leq k} I_{p}=\left\{i ; u_{i} \neq 0\right\} \text { and } \bigcup_{0 \leq p \leq k} I_{p}^{\prime}=\left\{i ; u_{i}=0\right\} .
$$

In conclusion, for an element $s$ in $\Xi$, we associate $u=\left(u_{1}, \ldots, u_{r}\right), k$ in $\{1, \ldots, r\}$, and the partition of the set $\{1, \ldots, r\}$ defined above. We also define for $1 \leq l \leq k$,

$$
u^{(l)}=\left(u_{i_{l}+1}, u_{i_{l}+2}+\frac{d}{2}, \ldots, u_{i_{l}+j_{l}}+\frac{d}{2}\left(j_{l}-1\right)\right),
$$

which is in $\mathbb{R}^{j_{l}}$ and the element of $\mathbb{R}^{r}$

$$
s^{(l)}=(\underbrace{0, \ldots, 0}_{i_{l} \text { terms }}, u^{(l)}, \frac{d}{2} j_{l}, \ldots, \frac{d}{2} j_{l}),
$$

which can be written as

$$
s^{(l)}=\left(\alpha_{1}^{(l)}, \ldots, \alpha_{r}^{(l)}\right)
$$

with

$$
\begin{cases}\alpha_{p}^{(l)}=0 & \text { if } 1 \leq p \leq i_{l} \\ \alpha_{i_{l}+p}^{(l)}=u_{i_{l}+p}+\frac{d}{2}(p-1) & \text { if } 1 \leq p \leq j_{l} \\ \alpha_{p}^{(l)}=\frac{d}{2} j_{l} & \text { if } \quad i_{l}+j_{l}+1 \leq p \leq r .\end{cases}
$$

The last term disappears if $i_{l}+j_{l}=r$. 
Proposition 3.5 With the previous notations, for any $s$ in $\Xi$, we have

$$
s=\sum_{1 \leq l \leq k} s^{(l)} .
$$

Proof. Recall that the corresponding vector $u=\left(u_{1}, \ldots, u_{r}\right)$ to a given $s$ in $\Xi$ is such that

$$
s_{1}=u_{1} \text { and } s_{i}=u_{i}+\frac{d}{2}\left(\varepsilon\left(u_{1}\right)+\ldots+\varepsilon\left(u_{i-1}\right)\right)
$$

Given $m$ in $\{1, \ldots, r\}$, we distinguish between four cases according to its position in the elements $I_{0}^{\prime}, I_{l}^{\prime}, I_{k}^{\prime}$ and $I_{l}$ of the partition of $\{1, \ldots, r\}$.

If $m \in I_{0}^{\prime}$, then $s_{m}=0$ and $\alpha_{m}^{(l)}=0$, for $1 \leq l \leq k$, since $m \leq i_{1} \leq i_{l}$ so that we have

$$
s_{m}=\sum_{1 \leq l \leq k} \alpha_{m}^{(l)}
$$

If $m \in I_{l}$ with $1 \leq l \leq k$, then $i_{l}+1 \leq m \leq i_{l}+j_{l}$. . It follows that

$$
\left\{\begin{array}{l}
\alpha_{m}^{(p)}=\frac{d}{2} j_{p} \quad \text { if } \quad 1 \leq p \leq l-1, \quad \text { since } i_{p}+j_{p} \leq i_{l-1}+j_{l-1}<i_{l}<m \\
\alpha_{m}^{(p)}=u_{m}+\frac{d}{2}\left(m-i_{l}-1\right) \quad \text { if } \quad p=l, \quad \text { since } 1 \leq m-i_{l} \leq j_{l} \\
\alpha_{m}^{(p)}=0 \quad \text { if } \quad l+1 \leq p \leq k, \quad \text { since } m \leq i_{l}+j_{l}<i_{l+1}<i_{p} .
\end{array}\right.
$$

Therefore

$$
\sum_{1 \leq p \leq k} \alpha_{m}^{(p)}=u_{m}+\frac{d}{2}\left(m-i_{l}-1+j_{1}+\ldots+j_{l-1}\right)=s_{m}
$$

If $m \in I_{l}^{\prime}$, with $1 \leq l \leq k-1$, then $i_{l}+j_{l}+1 \leq m \leq i_{l+1}$. Il follows that

$$
\left\{\begin{array}{l}
\alpha_{m}^{(p)}=\frac{d}{2} j_{p} \quad \text { if } \quad 1 \leq p \leq l, \quad \text { since } i_{1}+j_{1}<\ldots<i_{l}+j_{l}<m \\
\alpha_{m}^{(p)}=0 \quad \text { if } \quad l+1 \leq p \leq k, \quad \text { since } m \leq i_{l+1}<i_{l+2}<\ldots<i_{k} .
\end{array}\right.
$$

As $u_{m}=0$, we obtain

$$
\sum_{1 \leq p \leq k} \alpha_{m}^{(p)}=\frac{d}{2}\left(j_{1}+\ldots+j_{l}\right)=s_{m}
$$

If $m \in I_{k}^{\prime}$, then $i_{k}+j_{k}+1 \leq m \leq r$. Since $i_{1}+j_{1}<\ldots<i_{k}+j_{k}<m$, it follows that

$$
\alpha_{m}^{(p)}=\frac{d}{2} j_{p} \quad 1 \leq p \leq k .
$$


Thus

$$
\sum_{1 \leq p \leq k} \alpha_{m}^{(p)}=\frac{d}{2}\left(j_{1}+\ldots+j_{k}\right)=s_{m}
$$

To continue our description of the Riesz Mesures, we require some further notations. For $s$ in $\Xi$, and $1 \leq l \leq k$, where $k$ is the integer corresponding to $s$ defined above, we set

$$
\begin{aligned}
\bar{c}_{i_{l}} & =c_{i_{l}+1}+\ldots+c_{r} \\
\bar{c}_{i_{l}, j_{l}} & =c_{i_{l}+1}+\ldots+c_{i_{l}+j_{l}}
\end{aligned}
$$

$\bar{c}_{i_{l}, j_{l}}$ is an idempotent of rank $j_{l}$ in $V\left(\bar{c}_{i_{l}}, 1\right)$.

Let $\widehat{V}\left(\bar{c}_{i_{l}, j_{l}}, 1\right)$ and $\widehat{V}\left(\bar{c}_{i_{l}, j_{l}}, \frac{1}{2}\right)$ be the subspaces of $V\left(\bar{c}_{i_{l}}, 1\right)$ corresponding to the eigenvalues 1 and $\frac{1}{2}$, and let $\widehat{\Omega}_{\bar{c}_{i_{l}, j_{l}}}$ be the symmetric cone associated to $\widehat{V}\left(\bar{c}_{i_{l}, j_{l}}, 1\right)$.

Consider the map

$$
\alpha: \widehat{\Omega}_{\bar{c}_{i_{l}, j_{l}}} \times \widehat{V}\left(\bar{c}_{i_{l}, j_{l}}, \frac{1}{2}\right) \rightarrow V\left(\bar{c}_{i_{l}}, 1\right) ;(x, v) \longmapsto x+2 v \sqrt{x}+\left(\bar{c}_{i_{l}}-\bar{c}_{i_{l}, j_{l}}\right) v^{2},
$$

and let $i$ be the canonical injection of $V\left(\bar{c}_{i_{l}}, 1\right)$ into $V$.

We now define the measure

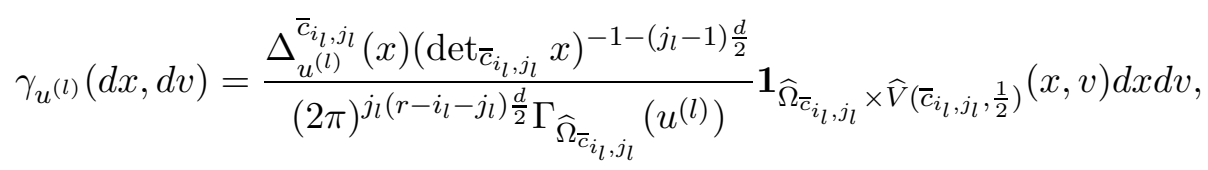

and we denote $\mu_{u^{(l)}}$ the image of $\gamma_{u^{(l)}}$ by the map $i \circ \alpha$.

We are now ready to state and prove our main result.

Theorem 3.6 For all $s$ in $\Xi$, we have

$$
R_{s}=\mu_{u^{(1)}} \star \ldots \star \mu_{u^{(k)}},
$$

where $\star$ is the convolution product.

Proof. We need to show that the Laplace transform of $\mu_{u^{(1)}} \star \ldots \star \mu_{u^{(k)}}$ defined in an element $\theta$ of $-\Omega$ is equal to $\Delta_{s}\left(-\theta^{-1}\right)$.

For $1 \leq l \leq k$, let $\theta=\theta_{1}+\theta_{12}+\theta_{0}$ be the Peirce decomposition of $\theta$ with respect to $\bar{c}_{i_{l}}$. If we denote $\mu_{u^{(l)}}^{\prime}$ the image of $\gamma_{u^{(l)}}$ by the map $\alpha$, then according to Theorem (3.2), we have that

$$
L_{\mu_{u}^{\prime}(l)}\left(-\theta_{0}\right)=\Delta_{s^{\prime}(l)}^{\bar{c}_{i_{l}}}\left(-\theta_{0}^{-1}\right),
$$


where $s^{\prime(l)}=\left(u^{(l)}, \frac{d}{2} j_{l}, \ldots, \frac{d}{2} j_{l}\right) \in \mathbb{R}^{r-i_{l}}$. On the other hand, as $\mu_{u^{(l)}}$ is the image of $\mu_{u^{(l)}}^{\prime}$ by the canonical injection of $V\left(\bar{c}_{i_{l}}, 1\right)$ into $V$, Theorem (3.4) implies that

$$
L_{\mu_{u}(l)}\left(\theta_{0}\right)=\Delta_{s}(l)\left(-\theta^{-1}\right),
$$

where $s^{(l)}=\left(0, \ldots 0, s^{(l)}\right) \in \mathbb{R}^{r}$.

Therefore the Laplace transform of $\mu_{u^{(1)}} \star \ldots \star \mu_{u^{(k)}}$ in $\theta \in-\Omega$ is

$$
\begin{aligned}
L_{\mu_{u}(1) \star \ldots \star \mu_{u}(k)}(\theta) & =\prod_{1 \leq l \leq k} L_{\mu_{u}(l)}(\theta) \\
& =\prod_{1 \leq l \leq k} \Delta_{s^{(l)}}\left(-\theta^{-1}\right) \\
& =\Delta \sum_{1 \leq l \leq k} s^{(l)}\left(-\theta^{-1}\right) \\
& =\Delta_{s}\left(-\theta^{-1}\right),
\end{aligned}
$$

which is the desired result

corollary 3.7 a) The measure $\mu_{u^{(l)}}$ is supported by the set

$$
\begin{aligned}
& J_{u^{(l)}}^{\prime}=\left\{x \in V\left(\bar{c}_{i_{l}}, 1\right) \text { such that } x \in \bar{\Omega} \quad \text { and rankx }=j_{l}\right\} \\
& \text { b) The measure } R_{s} \text { is supported by the set }
\end{aligned}
$$

$$
J_{u^{(1)}}^{\prime}+\ldots J_{u^{(k)}}^{\prime} \subseteq V\left(\bar{c}_{1}, 1\right) \cap \bar{\Omega}
$$

\section{Proof.}

a) Follows from Corollary 1.1.

b) It suffises to observe that $V\left(\bar{c}_{1}, 1\right) \supset V\left(\bar{c}_{2}, 1\right) \supset \ldots \supset V\left(\bar{c}_{k}, 1\right)$

Remark 3.1 a) When $s$ is in $\Xi$ such that $s_{i}>(i-1) \frac{d}{2}, \quad 1 \leq i \leq r$, then the integer $k$ corresponding to $s$ is equal to 1 . In this case $R_{s}=\mu_{u^{(1)}}$, it is concentrated on $\Omega$.

b) When $s$ is in $\left.\Xi \backslash \prod_{i=1}^{r}\right](i-1) \frac{d}{2},+\infty$, then the integer $k$ corresponding to $s$ is strictly greater than 1 and $j_{1}+\ldots+j_{k}<r$. The measure $R_{s}$ is in this case concentrated on $J_{u^{(1)}}^{\prime}+\ldots J_{u^{(k)}}^{\prime}$ whose the element are of rank less than or equal to $j_{1}+\ldots+j_{k}$. As $j_{1}+\ldots+j_{k}<r, R_{s}$ is supported by the boundary $\partial \Omega$ of the symmetric cone $\Omega$.

\section{References}

[1] J. Faraut, A. Korányi. (1994). Analysis on symmetric cones, Oxford Univ, Press.

[2] S.G. Gindikin. (1964). Analysis on homogeneous domains. Russian Math. 
Surveys. 29,) 1-89.

[3] A. Hassairi, S.Lajmi. (2001). Riesz exponential families on symmetric cones. J. Theoret. Probab. Vol 14, 927-948.

[4] A. Hassairi, S.Lajmi. (2004). Classification of Riesz exponential families on a symmetric cone by invariance properties. J. Theoret. Probab. Vol 17, No.3.

[5] M. Lassalle. (1987). Algèbre de Jordan et ensemble de Wallah. Invent. Math. 89, 375-393.

[6] H. Massam, E. Neher. (1997). On transformation and determinants of Wishart variables on symmetric cones. J. Theoret. Probab. Vol 10, 867-902. 